\title{
Orthogonal Netted Radar Systems
}

\author{
Hai Deng \\ Department of Electrical Engineering \\ The University of New Orleans \\ New Orleans, LA 70148-2215, USA
}

Abstract: - Orthogonal Netted Radar System (ONRS) is proposed to significantly improve radar performances by using a group of specially designed coding waveforms. The principle of ONRS is described. The possible approaches to design the coding waveforms for ONRS are introduced and some design results are presented.

Key-words: - Netted radar systems, radar signal design, waveform diversity, orthogonal signal set, matched filtering, simulated annealing

\section{Introduction}

An adaptive performance-enhanced radar system can be formed by netting multiple radar systems through a Central Processing Computer (CPC) [1-3]. Orthogonal Netted Radar System (ONRS) consists of multiple identical conventional radars stations that locate in different positions and operate with the same carrier frequency, and are controlled and coordinated by a CPC [46]. Each radar station in ONRS uses a coding radar waveform from a group of orthogonal coding waveforms in which a waveform is not correlated to any other waveform. Unlike the traditional netted radar systems, by employing orthogonal coding waveforms the radar stations in ONRS can operate at the same carrier frequency without mutual interferences and detection confusion. Hence, ONRS can adaptively function as a group of netted conventional monostatic radars, and as a multistatic radar system. For an ONRS with $N$ radar stations each of which can operate in monostatic and bistatic modes simultaneously, the maximum number of radar echoes from a target is $N^{2}$. Therefore, through information fusion processing ONRS will greatly improve the radar detecting and automatic tracking performances. Because ONRS can operate in bistatic or mulitstaic modes, it possesses much stronger capability in target recognition, anti-stealth, anti-ARM (Anti-Radiation Missile), and ECCM compared with a conventional radar system. The system is especially promising, if used the missile defense system, because it has to potential to accurately obtain the information of high-speed maneuvering targets. However, the key to the successful operations of ONRS is that the radar stations in the system need to transmit the orthogonal coding waveforms. The waveforms used by ONRS are defined as a coding waveform set in which each of the waveforms has the nearly ideal aperiodic autocorrelation property and any two of them have no cross-correlation. Therefore, the design of such waveforms is critical to the application of ONRS. 


\section{Waveform Design for ONRS}

If orthogonal coding waveform set used by ONRS consists of $L$ coding waveforms $\left\{s_{l}(t), l=1,2, \ldots, L\right\}$ with each waveform coded with $N$ subpulses, the following properties must be satisfied in the waveform design:

$$
\begin{aligned}
& A\left(s_{l}, \tau\right)=\frac{1}{N} \int_{t} s_{l}(t) s_{l}^{*}(t-\tau) d t \\
& =\left\{\begin{array}{ll}
1 & \tau=0 \\
0 & \text { otherwise }
\end{array} \quad l=0,1, \ldots, L-1\right.
\end{aligned}
$$

and

$$
\begin{aligned}
& C\left(s_{p}, s_{q}, \tau\right)=\frac{1}{N} \int_{t} s_{p}(t) s_{q}^{*}(t-\tau) d t \approx 0 \\
& \mathrm{p} \neq \mathrm{q}, \quad \forall \tau \in R, p, q=0,1, \ldots, L-1
\end{aligned}
$$

where $A\left(s_{l}, \tau\right)$ is the aperiodic autocorrelation function of $s_{l}(\mathrm{t})$ and $C\left(s_{p}, s_{q}, \tau\right)$ is the crosscorrelation function of $s_{p}(\mathrm{t})$ and $s_{q}(\mathrm{t})$.

The subpluse $s_{l}(\mathrm{t})$ in (1) and (2) can be binary, polyphase or frequency-hopping coded, and apparently each of the coding waveforms has nearly ideal noise-like autocorrelation property and any two of them have no crosscorrelation. Therefore, by using the waveform set, all radar stations in a netted system can transmit the waveforms simultaneously to the same target, with matched filtering processing each of the radar systems detects and picks up only the waveform transmitted by itself; the echoes from the other transmitters are not correlated to the matched filter at the receiver and thus are suppressed. If the receivers are equipped with multiple parallel matched filters, each radar station can operate in the multistatic mode to receive and process the echoes from the other radar stations as well as in the regular monostatic mode.

Algebraic construction methods have been widely used for the design of a coding sequence with good aperiodic autocorrelation property [7]. However, it seems to be very difficult to design a group of coding waveforms that possess the autocorrelation and cross-correlation properties as required in (1) and (2) by using algebraic methods. Alternatively, the numerical optimization is more favorable approach [8]. Since the numerical optimization of a code sequence is a NP-hard problem, the statistical algorithms such as Simulated Annealing or Genetic Algorithm have to be applied. To order to search the best waveform set with the properties in (1) and (2) numerically, one has to choose a cost function for the optimization. The cost function can be chosen either as the sum of the maximum sidelobe values of the autocorrelation functions and the maximum crosscorrelation function values $E_{1}$ or as the total autocorrelation sidelobe energy and cross-correlation function energy $E_{2}$. Therefore, the cost functions are:

$$
\begin{aligned}
& E_{1}=\sum_{j=1}^{L} \max _{m \neq 0}\left|A\left(s_{l}, m\right)\right|+ \\
& \lambda \sum_{p=1 q=p+1}^{L-1} \sum_{m}^{L} \max _{m}\left|C\left(s_{p}, s_{q}, m\right)\right|
\end{aligned}
$$

and

$$
\begin{aligned}
& E_{2}=\sum_{j=1}^{L} \int_{\tau \neq 0}\left|A\left(s_{l}, \tau\right)\right|^{2} \\
& +\lambda \sum_{p=1}^{L-1} \sum_{q=p+1}^{L} \int_{\tau}\left|C\left(s_{p}, s_{q}, \tau\right)\right|^{2}
\end{aligned}
$$

where $\lambda$ is the weighting coefficient between the autocorrelation function sidelobe and the crosscorrelation function in the cost function. The best coding waveforms for ONRS are 
obtained when the cost function in (3) or (4) is minimized.

\section{Design Results}

Some preliminary results are obtained for the design of binary coding waveform sets for ONRS by using Simulated Annealing (SA) algorithm through the minimization of the cost function in (4). SA algorithm suggests an analogy between the search for a minimum in a cost function and the physical process by which a material change state while minimizing its energy. The temperature is constantly reduced during the annealing process. At each temperature, the values of the binary coding sequences are randomly chosen and "perturbed," i.e. "+1" is changed to " -1 " and vice versa. If the cost function value is reduced as a result of code "perturbation", the new code is accepted; otherwise the new code is accepted with a probability of $\exp (-\Delta E / T)$, where $\Delta E$ is the cost change after the code "perturbation," and $T$ is the current temperature. The code "perturbation" is repeated until the cost values reach a pre-defined equilibrium state. Then the temperature is reduced and a new equilibrium state is reached at current temperature. The annealing process is stopped when the cost is not reduced during three consecutive temperature reductions. Table 1 is the designed binary coding waveform set

\section{References:}

[1] G. V. Trunk, "Automatic detection, tracking, and sensor integration," in Radar Handbook, $2^{\text {nd }}$ ed., pp. 840-848, McGraw-Hill, New York, 1990.

[2] V. S. Chernyak, Fundamental of multi-site radar systems: multistatic radars and multiradar consisting Code 1, Code 2 and Code 3 with code length of 40 and set size of 3 . The autocorrelation functions of Code1, Code 2 and Code 3 are shown in Figure 1 and the cross-correlation functions of them are displayed in Figure 2.

Even though the code length is relatively small, the autocorrelation functions appear to be thumbtack-like and the cross-correlations between the code sequences are weak. The results are fitted to the design requirements set in (1) and (2). Further numerical results show that the maximum sidelobe level of the autocorrelation and maximum crosscorrelation of the designed binary codes generally grow with a rate of $\sqrt{N}$. Figure 3 shows the matched processing results at three radar receivers of an ONRS with the waveform set in Table 1 applied. It is assumed that all three radar stations operate in monostatic mode simultaneously with the same target range and RCS.

The interferences from the autocorrelation sidelobes and crosscorrelations in Figure 3 can be removed through CLEAN algorithms. The other advantages to use ONRS include more accurate target measurements and efficient target recognition through multiple range profiles.

systems, Gordon and Breach Science Publishers, London, UK, 1998.

[3] W. G. Bath, "Association of multisite radar data in the presence of large navigation and sensor alignment errors," IEEE Int. Radar Conf., pp. 169-173, London, UK, 1982. 
[4] H. Deng, "Signal design and processing for orthogonal netted radar system," IEEE AP-S International Symposium on Antennas and Propagation, Columbus, Ohio, June 2003.

[5] H. Deng, "Polyphase coding signal design for orthogonal netted radar systems," Accepted. To appear in IEEE Transactions on Signal Processing.

[6] H. Deng, "Discrete-frequency coding waveform design for netted radar systems," IEEE Signal Processing Letters, vol.11, pp. 179-182, no. 2, February 2004.

[7] R. Turyn and J. Stover, "On binary sequences," Proc. Am. Math. Soc., vol. 12, pp. 394-399, June 1961.

[8] H. Deng, "Synthesis of binary sequences with good autocorrelation and cross-correlation properties by simulated annealing," IEEE Transactions on Aerospace and Electronic Systems, vol.32, pp. 98-107, Jan. 1996. 
Table 1 Designed ONRS Waveform Set with $N=40$ and $L=3$

\begin{tabular}{|c|c|c|c|}
\hline No. & Code 1 & Code 2 & Code 3 \\
\hline 1 & +1 & -1 & -1 \\
\hline 2 & -1 & -1 & +1 \\
\hline 3 & -1 & 1 & +1 \\
\hline 4 & +1 & -1 & -1 \\
\hline 5 & -1 & -1 & +1 \\
\hline 6 & +1 & -1 & +1 \\
\hline 7 & -1 & 1 & +1 \\
\hline 8 & -1 & -1 & -1 \\
\hline 9 & -1 & +1 & -1 \\
\hline 10 & +1 & -1 & +1 \\
\hline 11 & -1 & -1 & -1 \\
\hline 12 & -1 & -1 & -1 \\
\hline 13 & +1 & -1 & +1 \\
\hline 14 & +1 & +1 & +1 \\
\hline 15 & -1 & -1 & -1 \\
\hline 16 & +1 & +1 & +1 \\
\hline 17 & -1 & -1 & -1 \\
\hline 18 & -1 & -1 & +1 \\
\hline 19 & +1 & +1 & -1 \\
\hline 20 & +1 & +1 & +1 \\
\hline 21 & +1 & +1 & +1 \\
\hline 22 & -1 & +1 & +1 \\
\hline 23 & -1 & -1 & +1 \\
\hline 24 & +1 & +1 & -1 \\
\hline 25 & +1 & +1 & -1 \\
\hline 26 & -1 & -1 & +1 \\
\hline 27 & -1 & -1 & +1 \\
\hline 28 & -1 & -1 & +1 \\
\hline 29 & -1 & -1 & -1 \\
\hline 30 & -1 & -1 & +1 \\
\hline 31 & -1 & +1 & -1 \\
\hline 32 & +1 & +1 & -1 \\
\hline 33 & -1 & +1 & -1 \\
\hline 34 & -1 & +1 & -1 \\
\hline 35 & -1 & +1 & +1 \\
\hline 36 & -1 & +1 & +1 \\
\hline 37 & -1 & -1 & +1 \\
\hline 38 & -1 & +1 & -1 \\
\hline 39 & -1 & +1 & +1 \\
\hline 40 & +1 & -1 & -1 \\
\hline
\end{tabular}



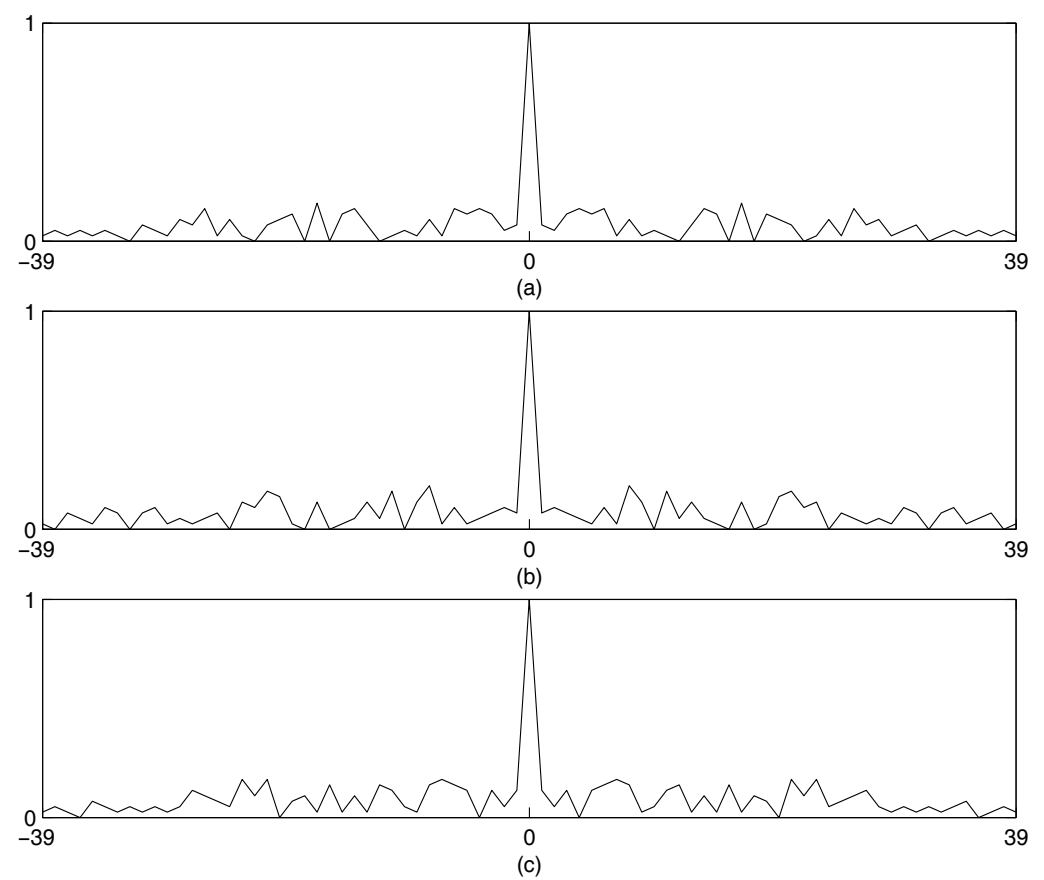

Figure 1 The autocorrelation functions of (a) Code 1, (b) Code 2 and (c) Code 3 in Table 1
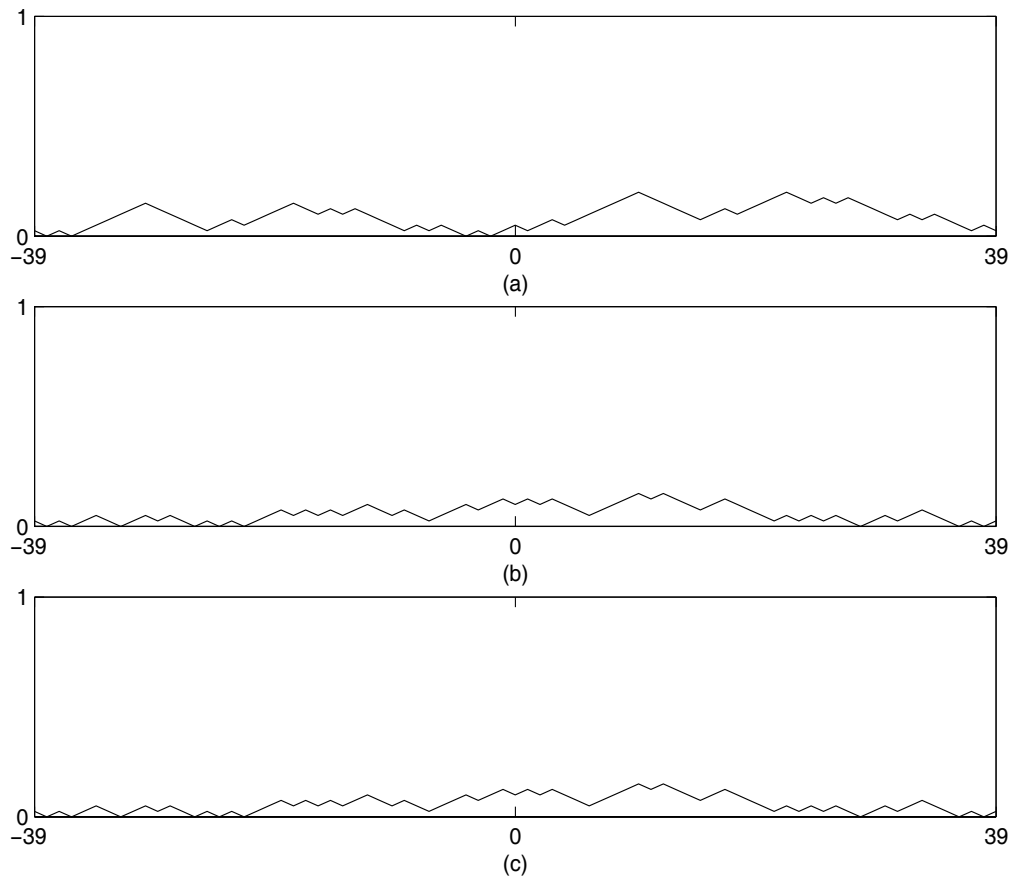

Figure 2 The cross-correlation functions of (a) Code 1 and Code 2, (b) Code 1 and Code 3 and (c) Code 2 and Code 3 


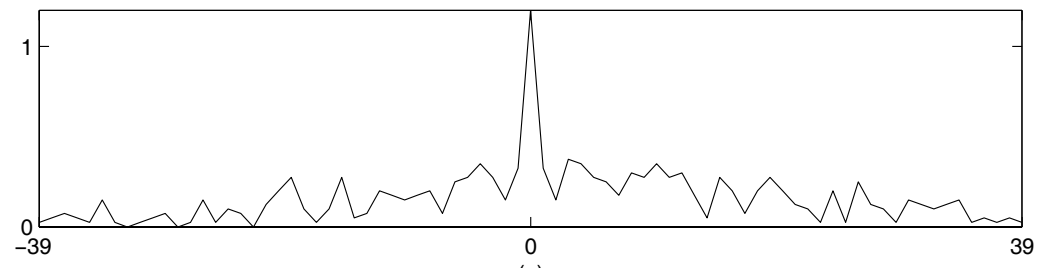

(a)
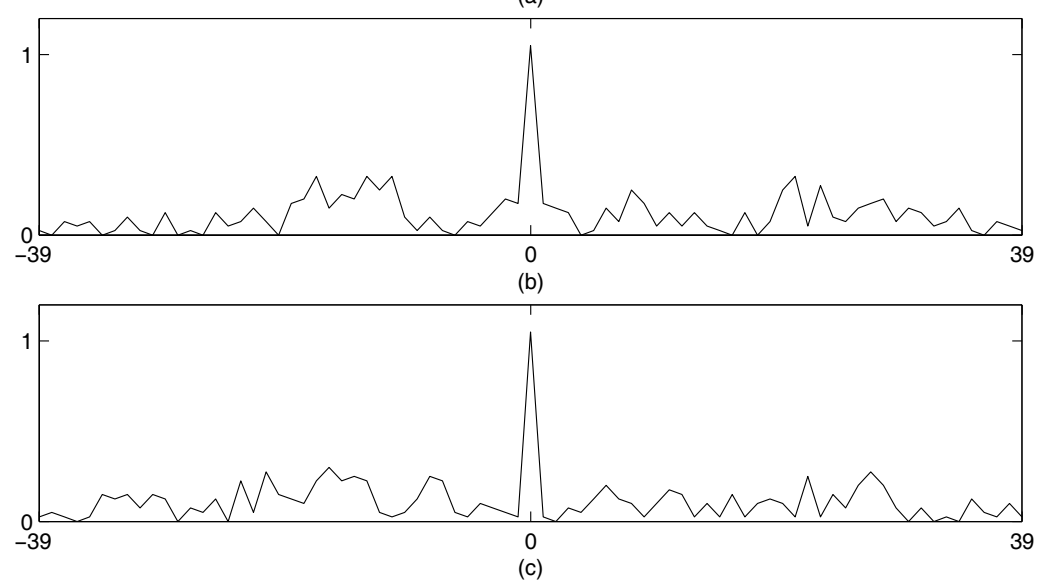

Figure 3 The Matched Filtering Outputs of ONRS Receivers for a Target with the Waveform Set in Table 1 Applied 